\title{
On the Significance of the Identity Debate in DBS and the Need of an Inclusive Research Agenda. A Reply to Gilbert, Viana and Ineichen
}

\author{
Anke Snoek (D) - Sanneke de Haan • \\ Maartje Schermer • Dorothee Horstkötter
}

Received: 29 January 2019 / Accepted: 6 May 2019/Published online: 26 May 2019

(C) The Author(s) 2019

\begin{abstract}
Gilbert et al. (Neuroethics, 2018) argue that the concerns about the influence of Deep Brain Stimulation (DBS) on - as they lump together - personality, identity, agency, autonomy, authenticity and the self (PIAAAS) are due to an ethics hype. They argue that there is only a small empirical base for an extended ethics debate. We will critically examine their claims and argue that Gilbert and colleagues do not show that the identity debate in DBS is a bubble, they in fact give very little evidence for that. Rather they show the challenges of doing research in a field that is stretched out over multiple disciplines. In that sense their paper is an important starting point for a discussion on methodology and offers valuable lessons for a future research agenda.
\end{abstract}

A. Snoek $(\bowtie) \cdot$ D. Horstkötter

Department of Health, Ethics and Society, School for Mental Health and Neuroscience, Maastricht University, P.O. Box 616, 6200 MD Maastricht, the Netherlands

e-mail: a.snoek@maastrichtuniversity.nl

D. Horstkötter

e-mail: d.horstkoetter@maastrichtuniversity.nl

\section{S. de Haan}

School of Humanities and Digital Sciences, Culture Studies, Tilburg University, Warandelaan 2, Tilburg, the Netherlands e-mail: Sannekedehaan@gmail.com

M. Schermer

Department of Medical Ethics and Philosophy, Erasmus MC, PO Box 2040, 3000 CA Rotterdam, the Netherlands

e-mail: m.schermer@erasmusmc.nl
Keywords Autonomy· Agency $\cdot$ Deep brain stimulation $\cdot$ Identity $\cdot$ Neuroethics $\cdot$ Self . Multidisciplinary research $\cdot$ Interdisciplinary methodology

\section{Introduction}

In their review study, Gilbert, Viaña, and Ineichen [1] argue that the ethical concerns about the influence of Deep Brain Stimulation (DBS) on (as they lump together) personality, identity, agency, autonomy, authenticity and the self (PIAAAS) are due to a bubble that need to be deflated. They point towards the comparatively low number of primary studies conducted to date, that are greatly outnumbered by ethical studies. The question is whether ethicists do not make too much out of these very few examples of personality changes following DBS: that one patient may have felt like an electric doll [2] should not entice us to jump to conclusions about the effects of DBS in general. Against that background, they make an urgent request for more primary studies to better substantiate current ethical debates and the serious concerns expressed about the implications of DBS. So far, we agree with their findings: there is a small empirical basis for a large neuroethical debate, and more empirical research will result in a better informed neuroethical debate, and better informed patients, family and clinicians.

However, Gilbert et al. do not only point to the asymmetry between the small portion of empirical studies and the booming ethical literature. They also suggest that the ethical literature is a bubble and that it 
exaggerates the influence of DBS on PIAAAS. They end up being quite dismissive about the role of ethicists in the debate, and the scientific status of qualitative research. They dismiss case studies as anecdotal evidence, and argue for more standardized measurements. They sketch an image of neuro-ethicists as either being ignorant about how to properly address empirical findings, or being tweaking empirical evidence to support their own pet projects. They argue that the debate needs less ethics involvement, and more standardized measurements of PIAAAS change (their arguments largely echo those of Müller, Bittlinger, and Walter [3]).

In the following, we will react to and comment on Gilbert et al.'s arguments and thoughts. In the second and third sections we will discuss their claims that there is very little evidence that DBS can cause changes in PIAAAS and that ethicists use empirical findings selectively, or not at all. In the fourth section we will look into their unfortunate choice to not define what they understand by PIAAAS changes, and when or why such changes are problematic. In the fifth and sixth sections we will critically examine their plea for more standardized measurements, and we will outline the added value of ethicists and qualitative research next to quantitative research. Finally, in the seventh section, we will sketch the contours of a multidisciplinary research agenda needed to properly examine PIAAAS changes after DBS.

\section{Is there Little Evidence that DBS Influences PIAAAS?}

Gilbert and colleagues make two slightly overlapping claims. The first claim is that there is very little proof for PIAAAS changes due to DBS. Their second claim is that ethicists use the scarce empirical literature not at all, selectively, or take it out of context and exaggerate the empirical evidence. For both claims they provide very little evidence.

Let us first look at their first claim: that there is little proof of PIAAAS changes after DBS treatment. Gilbert et al. argue that the occurrence of such changes seems rare and point out that if they occur it is unclear whether they are actually due to DBS.

Having reviewed empirical literature, they claim that a majority, that is, 43 out of 64 articles (67\%), 'do not support direct links of DBS on PIAAAS' (p.8). These 64 articles are what they call 'primary studies', that is: 'new reports of clinical cases involving first-hand interviews or clinical studies involving implanted patients' (p. 3 our italics). These are, however, different types of studies. No information is given on those 43 papers that are supposed to count as evidence that the idea that DBS causes personality changes is a bubble. But to estimate their quality as evidence we need to know more details about the studies. What kind of studies are they? Were patients being interviewed and if so, were they asked explicitly about PIAAAS-type changes? Or were these rather medical studies referring to symptom scales and did any PIAAAS-type changes only have a chance of showing up as a side-effect? Were PIAAAS-type changes a central concern in these studies? How were such changes defined in these articles? Were they even defined at all? And how many of these 43 studies were sponsored by DBS manufacturers like Medtronics? As long as these 43 papers remain a black box, it is not clear what conclusions we can draw from them. It is especially difficult to weigh their apparent rejection of the idea that DBS causes PIAAAS-type changes against the 8 studies that do report PIAAAStype changes in patients (with all necessary nuances and cautions that need to be observed regarding these reports). In Gilbert et al.'s overview of empirical studies that do report PIAAAS changes, Gilbert et al.'s own study [4] and De Haan et al. [5] are missing, which both claim that DBS could lead to PIAAAS changes. ${ }^{1}$ Gilbert and colleagues also write that 'the number of reported (putative) DBS-induced PIAAAS changes appears to be extremely low', given that only a part of 168 patients out of approximately $150.000 \mathrm{~dB}$ patients reported PIAAAS-like changes (p.9-10). But that is of course a highly misleading picture. These 168 patients were asked about their experiences - and we do not know whether any of the other 149,832 patients experienced PIAAAS changes, because they were not asked about it. The number of patients that are researched and reported upon will always be smaller than the total population. To compare: how many patients were covered by these other 43 articles? And were they asked explicitly about PIAAAS type changes? We do not know. The lack of evidence should however not be taken as a sign of an evidence of lack, as Gilbert and colleagues also point out. In fact what we do know so far is that if we ask patients and their family members explicitly, some of them will indeed report PIAAAS-like changes. Another way to approach the question of prevalence could be to

\footnotetext{
${ }^{1}$ These articles are within their data search range.
} 
indicate what percentage of the 168 respondents reported PIAAAS change. In their own study for example, Gilbert and colleagues found that $47 \%$ of the respondents reported some form of self-estrangement. When extrapolated, this might mean that in total about 70.500 patients experience some sort of PIAAAS change.

As the authors themselves admit in their 'limitations' section, their choice of search-string to look into the empirical evidence may have been badly chosen. Medical papers reporting on the effects and side effects of DBS are not very likely to use terms like personality, identity, agency, autonomy, authenticity or self (PIAAAS), so using those terms as search-terms will lead to the exclusion of a great many potentially relevant articles. Medical papers may report, for example on 'hypomania', which may well lead to disinhibited behavior, impulsivity and even incompetence to decide (as in $[6,7]$. This may not be labelled as PIAAAS by the medical community, but it is a very relevant fact for ethicists discussing the impact of DBS on autonomy and authenticity. Medical papers may report on 'adaptation problems' or 'psycho-social problems' whereas a philosopher may call this a disruption of narrative identity [8], or of self. Similarly, Gilbert and colleagues refer to a paper by De Haan and colleagues [9] saying that it presents "no direct conclusion in relation to PIAAAS'. The paper, however, reports how OCD patients' (i) self-experiences, (ii) relation to the world, and (iii) reflective attitudes change following DBS. The paper also discusses whether these changes are best understood in terms of changes in the sense of self, or changes in agency, or changes in mood, and advocates that all of these are at stake and that we rather need a holistic perspective on what changes for patients after being treated with DBS. It therefore describes these changes as amounting to 'a different way of being in the world' - making for are a pretty clear case of changes in the PIAAAS-realm. These examples show how difficult, yet crucial it is to define what is meant by PIAAAS changes due to DBS. We will get back to this issue in the third section.

Apart from their claim about the relative rarity of PIAAAS changes following DBS, Gilbert and colleagues also point out that it is often hard to establish what causes the PIAAAS changes and thus to be sure whether they are really due to DBS specifically. This is a valid caution - and one that has been made by most of the primary studies in their Table 1, as well as in many conceptual papers.
Which changes are the effect of DBS and which are rather the effect of a decrease of symptoms of the disorder, or are a secondary effect even of these decreased symptoms (often called 'adjustment problems')? Besides, it is important to note that DBS treatment often comprises more than DBS alone. In order to optimally adjust the settings of the stimulator, patients are typically seen once a week or twice a month, especially in the beginning of treatment. Patients are asked how they are and are monitored carefully by a specialized team. In case of OCD, for instance, most patients also engage in Cognitive-Behavioural Therapy to get a grip on their compulsions. This seems to be an important part of the effect of treatment [10]. Summing up, we need to take into account that there is 1) a disorder (PD, OCD, addiction), which often already influences PIAAAS as well $[5,11]$. And 2) there might be underlying disorders that are masked by the primary disorder. Then there is 3) the direct influence of DBS on the brain, and there are 4) the indirect effects of DBS such as the recovery and the consequent 'biographical disruption' as discussed by Gisquet, Schechtman and Gilbert, plus 5) there are the other elements to DBS treatment. The question is what counts as causality: is it only (3), the direct influence of DBS on the brain, or also (4), the indirect effects, and do we also include the desired effect of DBS on the disorder as PIAAAS changes or only those that have a disrupting effect?

The implicit assumption Gilbert and colleagues seem to make, is that change should be mono causal, in order for DBS to be ethically problematic. But the case of DBS is of course more complex; making it hard to establish mono-causality - all the more so in empirical studies that are not specifically designed to do so. Gilbert et al. are too quick to dismiss DBS as a factor that influences PIAAAS, and hence being ethically relevant, because a simple mono-causality cannot be established $[12,13]$.

\section{Are Ethicists Misquoting the Empirical Literature?}

With regard to their second claim Gilbert et al. again give very little information about search terms, the selection process, or the analysis of the papers when reviewing the 'theoretical neuroethics papers'. We do not learn which or how many papers they reviewed. This makes their claims and conclusions non-verifiable. The tables they added unfortunately provide no useful information in this regard. 
Moreover, it remains unclear to what extent these 'theoretical neuroethics papers' really mis-quote, mis-interpret, or overstate empirical evidence, and, maybe even more important: to what extent the arguments and claims made in those papers actually depend upon empirical evidence. Some conceptual arguments can very well be made without a need for empirical evidence and casereports may be used in a merely illustrative or heuristic way. In the absence of a clear description of the claims and arguments made in the 'theoretical neuroethics papers' it is hard to assess whether these papers indeed base their arguments on insufficient evidence (more about this in the section 'The Role of Ethicists').

Let us look in more detail at two studies that they criticize as contributing to an ethics hype without empirical foundation: the studies of Baylis and Schermer $[14,15]$. Gilbert and colleagues claim that in interdisciplinary research, there is always a risk that one discipline takes evidence from another discipline out of context. For example, ethicists might cherry pick from empirical quotes (see also Müller, Bittlinger, and Walter 2017). Again, they give very little proof that this indeed happens, they just state that 'many neuroethicists and philosophers selected specific quotes' (p. 4), without giving an indication of which and how many studies and how these misuse the selective quotes.

They give one example of a quote that they consider misused. This quote comes from an empirical study by Schüpbach and colleagues [2], in which one of their respondents exclaims: 'I feel like an electric doll' (page 1813). They argue that this quote is wrongfully used by Baylis [14]. Although Baylis sees the quote as supporting evidence that DBS causes a threat to agency, Gilbert and colleagues stress that in the original study this quote has been given as evidence for an altered body image, but not as a change in PIAAAS and hence that ethicists who still use it in that sense make undue use of the primary source. Although Gilbert et al. are correct to point out the topic of change in body image, they are incorrect in taking that as an argument that therefore PIAAAS change would not apply. Apparently, they hold the assumption that our bodies and our agency are separate from each other. Nothing less is true, however. Instead, our bodies are very intimately related to our agency, and the kind(s) of agency we are capable of $[16,17]$. This relationship between body and agency becomes especially apparent when the body stops functioning the way it used to do [17-20], and hence potentially again when it regains lost capacities. In her paper
Baylis [14] explicitly discusses the complex relationship between self, body, identity and agency, using a firsthand account of a PD patient who received DBS treatment, whom she quotes extensively (i.e. Helmut Dubiel, a well-known German sociology professor who also wrote about his own experiences [21]). To simply assume that body image and agency are separate matters as Gilbert and colleagues do, is begging the question.

In trying to find evidence of ethicists making unfounded assumptions, Gilbert and colleagues often read selectively. Another example in this regard is their critique of Schermer's paper [22]. They argue that Schermer warns for implantable devices, but that she does not support this with empirical evidence, rather, she cites Hansson [23], whose paper they also dismiss as not being primary research. However, neither Hansson's paper nor Schermer's paper are about DBS, and neither claims that DBS causes PIAAAS. Hansson just points out - and Schermer quotes him on that - that new technologies that involve implants in the brain (Hansson here refers to cell-based implants, as well as to naturally occurring brain-changes such as tumor growth), raise questions about the concepts and distinctions we use, e.g. about the meaning of personal identity or personality change. The whole point of Schermer's quote, read in the context of the paper, is to say that new technologies can raise conceptual questions. The paper deals with those questions, it does not deal with alleged PIAAAS changes caused by DBS. Although she uses the casereport of Leentjens et al. to illustrate a theoretical point about responsibility, she nowhere claims that hypomania or disinhibition - as described in Leentjens - are common or frequent side-effects of DBS [6].

\section{The Need to Define Rather than Lump Together PIAAAS Changes}

Gilbert and colleagues do not define what they understand by PIAAAS changes due to DBS. However, in their introduction they cite a study that compares the alleged impact of DBS to the case of Phineas Gage, whose left frontal lobe got pierced by a large iron rod during an accident. After this, his personality and behaviour radically changed [24]. It seems that Gilbert and colleagues have this kind of change in mind when they contest the PIAAAS-change claim. However, the Phineas Gage case seems to be the most radical idea about personality change due to tampering with the 
brain, and contrary to what Gilbert et al. suggest, we doubt that most ethicists have this in mind when talking about personality change due to DBS (see also [25]). ${ }^{2}$

Gilbert et al. seem to suggest that there is something like 'PIAAAS' in the neuroethics literature. But PIAAAS is their way of putting together quite different concepts. If you look at PIAAAS changes in general, then the terminology is indeed lose, but if one looks at the different neuroethics articles individually, one can see that most authors carefully describe the aspect of the PIAAAS conglomerate that they are interested in, which is often strongly grounded in a philosophical tradition, rather than 'serving the authors' philosophical accounts'.

Gilbert and colleagues acknowledge that the concepts of personality, identity, agency, autonomy, authenticity and self (PIAAAS) are complex constructs that are not easily measured empirically. However, if they do not specify which concept from the PIAAAS conglomerate a particular neuro-ethicist or philosopher is after, how can they state in general that DBS would not influence any of these concepts? Each of the notions that make up PIAAAS - personality, identity, agency, authenticity, autonomy, and self - are already subject of extensive debates on how to best define them. And since different researchers can mean different things by these notions, it is all the more crucial to specify how such PIAAAS changes are defined, to avoid talking at cross purposes. There are more and different ways to understand PIAAAS changes than just the Phineas Gage case (see also [13, 26]).

Take for instance, Schechtman's study [8], which the authors cite as being part of the ethics hype that exaggerates PIAAAS changes. When we look at her claims in more detail, we see that they are actually quite modest. Schechtman is interested in narrative identity - the stories people construct about themselves and their lives - which constitutes who they are as human beings. She opposes this concept of 'narrative identity' to 'numerical identity' which refers to being numerically the same person and argues that while DBS might interfere with a person's narrative identity it is of course numerically still the same person. Still, DBS can disrupt a person's narrative identity, meaning that people face significant difficulties in making DBS treatment a coherent part of

\footnotetext{
$\overline{2}$ Gilbert and colleagues state: 'Many publications suggest that when "DBS is applied to enhancing or maintaining movement, the specter of Phineas Gage, whose personality changed so radically after his brain was pierced by a tamping iron, haunts us. [DBS] may fundamentally alter selves"' (page 1).
}

the narratives about their lives. Although Schechtman does not cite her, Gisquet's [27] empirical study supports this ethical concern, in that it describes how DBS for PD can constitute a unique biographical disruption. Several patients expected to return to their pre-disease state after DBS. Regarding their movement capacities, this indeed occurred. However, regarding their social relationships and activities this expectation was not met in equal measure. Their activities and social relationships had been determined by their PD for years, and could not instantaneously switch back to the pre-PD state. Hence, DBS can lead to significant biographical and narrative disruptions, in which people experience significant difficulties to adapt to their post-DBS life. Such changes are definitely real and meaningful for patients. Ironically it was Gilbert himself who previously emphasized such disruptions under the denominator of 'burden of normality' [28].

While Gilbert et al. rightly state that PIAAAS concepts are hard to operationalize, their conclusion (p.11) that therefore they should remain in the domain of philosophical instead of empirical research is most unfortunate. What we rather need is more and richer data on the experiences of patients and their family to see how we can best operationalize PIAAAS change (see also [14]). Ideally, (qualitative) empirical research and conceptual considerations go hand in hand, as we will discuss later on.

Tightly connected to the question of how to define each of the elements of PIAAAS, is what counts as a change, and when and why any such changes would be problematic.

Let us look now at the second question, how much of an alteration or which kind of change of PIAAAS is needed in order to say that DBS affects these phenomena? For example, is the self-estrangement people experience post DBS, an effect Gilbert and Viaña found in their own empirical study [4], enough to count as a PIAAAS change? Or did Gilbert and Viaña leave out their own study of the review because they found the effect of self-estrangement to mild to count as a PIAAAS change?

Gilbert and colleagues argue that identity is a fluid concept: we constantly change throughout our lives. However, these are exactly the kind of questions philosophers try to illuminate: when is an identity change disruptive, and do changes for the better automatically feel as 'becoming oneself', and changes for the worst as 'losing oneself'? Especially in the case of DBS for 
psychiatric disorders, the philosophical identity debate is highly relevant. For psychiatric disorders affect how one feels, perceives, and/or acts: aspects that are typically felt to be closely related to one's sense of self. A broken leg does not affect my sense of who I am, but if I feel profoundly different (e.g. depressed or anxious) for an extended period of time, this does affect me as a person. And I can wonder if these feelings are a reflection of me, or rather the effects of a disorder. Since treatment of psychiatric disorders is also targeted at patients' feelings, thoughts, and actions, the question of how treatment affects patients' self is relevant in general. This is all the more complicated in case of psychiatric disorders with an early onset, such as OCD, in which there is no developed 'pre-disorder personality' to take as a comparison. There is no baseline, for patients simply do not know what kind of persons they would have been if they had not developed OCD at age nine. Patients (and their loved ones) face the sometimes difficult question which of the changes do belong to them and which of them are rather unwanted side-effects. For instance, when is an increased libido alienating? Before treatment, sexual desires would typically be far removed for patients, as their lives were taken up by surviving. But hypersexuality can be a side-effect of DBS treatment [29, 30]. When is your sex drive too much to fit you? Claiming that the identity debate is a hype does not further our understanding of these issues. Fruitful interdisciplinary collaborations, however, can.

Many of these conceptual questions on what change is, are already explicitly addressed in both the ethical and empirical studies that Gilbert and colleagues refer to. For instance, both Hansson's [23] paper and many of Schermer's papers $[15,31,32]$ make the same points Gilbert et al. make about e.g. the effects of the disease itself, of adaptation-problems related to recovery and the complexities of concepts such as PIAAAS. Especially Schermer [22] calls for conceptual research on such complex and often diffuse notions like PIAAAS, in the context of claims about 'personality changes' due to - in this case: cell-based - interventions in the brain. Most empirical studies address these questions too: De Haan and colleagues [33] for instance discuss the difficulties of defining personality change, the problem of which changes are problematic, and the difficulty of establishing the precise role of DBS in these changes. And Agid and colleagues [34] and Schüpbach and colleagues [2] provide thoughtful considerations on the role of indirect effects of DBS.

\section{The Role of Ethicists}

Gilbert and colleagues give an extended critique on the ethical literature: the attention to identity questions in DBS is out of proportion, the literature is too speculative, ethicists are indulging in conceptual discussion that lack empirical ground. Instead, they make a case for more empirical research, and more standardized ethics research.

We agree with Gilbert and colleagues that more empirical ethics research will enrich the debate. More empirical research will result in a better informed neuroethical debate, and better informed patients, family and clinicians. We agree that research focusing on standardized measurements can have additional value. What we contest is Gilbert et al.'s hierarchical view on the sciences. Each scientific method (ethics, philosophy, case studies, qualitative in-depth studies, quantitative studies, randomized controlled trials) has its own merits and weaknesses. And within each method, there will be poorly conducted studies, and superb studies. But in order to fully profit from the wide range of evidence that is available to us, we need to take each other's disciplines seriously. Multidisciplinary research thrives by equality and cooperation, not by hierarchy. Sure, we can imagine studies that are badly designed and conducted, but to be dismissive of a whole discipline, because it includes bad apples, is futile and cannot further the debate. Besides, as we discussed earlier, Gilbert and colleagues do not convincingly show to what extent, and how ethics literature misinterprets the empirical data. Rather than unmasking a bubble that needs to be deflated, Gilbert et al. unwittingly testify of the many challenges of doing research that stretches over multiple disciplines.

The first critique they have, is that there is only a small empirical basis for an extended ethical debate of PIAAAS. That is right, there is indeed a quantitative asymmetry between ethical studies and empirical studies, and many ethical discussion rely on only a few cases. Gilbert and colleagues, however, are very quick in attributing this phenomenon to an ethics hype. But there is a different way of considering this mismatch. On the one hand, this asymmetry simply points to a lack of empirical studies to date that explicitly target PIAAAS like changes. If there were more empirical data available, the mismatch would not be so large. On the other hand, the significant interest of ethicists in the effects of DBS does not necessarily mean that they are hungry for 
hypes: it is more likely that their interest in the possibility that a brain implant can affect people's sense of self is much more intrinsic. For with invasive brain modulation something novel seems to have entered the medical arena. ${ }^{3}$ Neuro-interventions trigger and raise ethical questions that are not triggered by other biomedical technologies ([38]). Hence, the rise of a great number of ethical studies following only a few primary studies is not as out of proportion as Gilbert et al. advocate.

We already argued that Gilbert et al's claim about the prevalence rates of PIAAAS type changes is unfounded. But even if these rates turned out to be low, that would not imply that any ethical concerns could therefore be dismissed. Prevalence rates are of a different order than ethical concerns. If the situation in only a handful of cases is sufficiently serious, this can definitely constitute a justified foundation for far-reaching ethical concerns. If you have a $1 \%$ chance to go blind with a certain medicine, you will be more reluctant to try it than if you have a $1 \%$ change to get temporary diarrhea. Dismissing ethical studies as constituting a bubble, because they would be underpowered so to say, falls short of understanding what the very point of an ethical analysis is (see also [36]). We do not infer that prevalence of a side effect has no bearing on ethical concerns; we just state that prevalence rates are of a different order than ethical concerns.

The second critique Gilbert and colleagues have on ethical studies, is that they are too speculative because they do not engage well enough with the empirical literature. In the first section we argued that they do not convincingly show to what extend the ethics papers misinterpret the empirical claims, nor to what extent an ethics paper relies on empirical data. Now, we want to further nuance their findings: the mismatch between empirical research and ethics is not as great as they assert. Of the eight top empirical studies they identify, three major ones have been published not earlier than 2015. So, they are relatively novel and could not have been taken into account when the boom of theoretical studies started to emerge in 2009. When there is empirical literature, ethical research should engage with it, but in the absence of empirical literature, the role of ethicists

\footnotetext{
${ }^{3}$ As the Nuffield Council on Bioethics aptly put it [35], our brain has a special status that distinguishes it from other organs. "Its healthy functioning plays a central role in the operation of our bodies, our capacities for autonomous agency, our conceptions of ourselves and our relationships with others - and thus in our abilities to lead fulfilling lives" (p. xix).
}

is to make conceptual claims. Ethicists have the difficult task of anticipating the ethical consequences of rapidly developing technologies. They have to engage in proactive reflection and should ideally think one or more steps ahead of these technologies in order to prevent damage before it is done. In that sense, what might seem to be the speculative character of ethics is a core value rather than a shortcoming.

\section{The Role of Qualitative Research}

Gilbert et al. are also quite skeptical about the use of case-studies and qualitative research in general. They argue that case-studies are merely providing anecdotal but not sufficiently robust evidence. And a major critique they have on both case-studies and qualitative research is that they fail to offer insight in prevalence rates. Gilbert and colleagues fear that qualitative research easily gets misinterpreted. Therefore, they advocate a more standardized, quantitative way of measuring PIAAAS change after DBS, since they think this will be more objective. In plain terms, they state that there should be more standardized measurements and randomized controlled trials; and they call for more reliability, robustness, and validity in the research around DBS and PIAAAS (page 13).

We are quite skeptical about this hierarchy of sciences Gilbert and colleagues proclaim, with certain medical methods on top and qualitative research and ethical analysis at the bottom. Qualitative and quantitative types of research methods have different strengths and weaknesses and perform different roles in the scientific process - as do philosophical and theoretical considerations. Quantitative research requires reliable scales, and as the authors point out, these have not yet been sufficiently developed for such intricate issues as personal identity and authenticity. One could wonder if such measures will ever be sufficiently developed, given that it is so easy to talk cross purposes; to mean different things even by using the same word. We found for instance that the question whether patients felt they had 'changed as a person' could either be understood in terms of behaving differently (in which case most patients would agree), or rather in terms of their values and beliefs (in which case patients did not feel changed). So a simple 'yes' or 'no' to this question on a questionnaire will not tell us much $[5,33]$. 
In our opinion, the place to start is precisely qualitative research combined with philosophical investigation. For in order to be able to quantify something, you should first have an idea of what it is that you want to quantify. Before we can measure things, we first need to define them - if only in a preliminary way [15]. That is: before we can answer the question of prevalence, we need to understand the nature of the effects whose prevalence we are attempting to measure. Which changes do amount to personality changes? Or maybe: which changes amount to problematic personality changes? And who gets to decide what counts a being 'problematic changes': patients only, or their loved ones too? And what if they disagree? Qualitative research, such as indepth interviews and case studies of salient cases, contribute to the scientific process precisely by addressing this very first question: what is happening in patients' lives, what do they experience, what do their partners, family members, or friends experience after DBS treatment? Qualitative research ideally precedes quantitative research. Likewise, theoretical considerations have their proper place in this scientific process too. Philosophical and ethical analyses of what we mean by 'PIAAAS'type changes is just as crucial. After all, the questions 'what is it that we are currently measuring' and 'what is it that we should be measuring' cannot be answered by any empirical data. To assume we could have data without concepts is an illusion, one that only leads to poorer research, built on implicit assumptions rather than proper hypotheses.

Case studies can be particularly suitable to understand PIAAAS change, because they can thoroughly explore one person's experience. Case-studies also reduce the risk of out of context quotes and are therefore well able to meet one of the concerns central also to Gilbert et al's arguments. Of course we should hold studies against a quality measure, and of course it can be complicated how evidence from one discipline is used in another. In general, philosophers are not well trained in ranking evidence, and performing literature reviews in evidencebased medicine style. These skills could be a valuable addition to their methodologies. But these issues are far from new, and are already extensively addressed in ethical literature as well (see for example [37]).

Gilbert et al's call for more standardized measurements and RCTs, their characterization of case studies as mere anecdotal evidence, and their dismissive tone about the role of theoretical ethicists disregards above features of interdisciplinary research and dialogues between the disciplines concerned. It risks muting the different voices, including the voices of patients, whose phenomenological experiences are seminal, not anecdotal, in disentangling the influence of DBS on PIAAAS.

\section{Take Home Message: the Future Agenda for DBS PIAAAS Research}

We have argued that Gilbert, Viaña, and Ineichen [1] failed to show that ethics literature exaggerates changes in personality, identity, agency, autonomy, authenticity, and the self (PIAAAS) after deep brain stimulation. There is not necessarily a low prevalence of such changes, but rather an unknown prevalence. Besides, even a low prevalence can still have a high clinical and theoretical relevance. Gilbert and colleagues have, rather, identified some of the challenges of doing research in an inherently multidisciplinary field.

These challenges regard how we define concepts, how we translate them across fields, and how we understand each other's methodology and findings. Gilbert and colleagues risk regressing the debate by proposing a hierarchy of sciences with certain quantitative methods on top and qualitative research and ethical analysis at the bottom. This proposition is detrimental for the multidisciplinary research that is much needed in this domain.

Multidisciplinary research can only thrive when different disciplines are being perceived as equally valuable, and as being valuable in their own way. Different disciplines should be complementary to each other instead of regarding them as opposed. Only then can we benefit from the different, unique perspectives that different disciplines have to offer.

Gilbert and colleagues lump together a wide range of concepts: personality, identity, agency, autonomy, authenticity, and the self. But what we rather need are proper definitions of these concepts, and a discussion on which changes are problematic and why, and who gets to define that. For that, we need the combined efforts of philosophers, qualitative and quantitative researchers, and of course patients and their loved ones.

Acknowledgments The authors would sincerely like to thank the two anonymous reviewers, who had great integrity, and provided some of the most engaged comments that we ever had from reviewers. For Sanneke de Haan this work is part of her research programme 'Authenticity in psychiatry' with project number 27520-067, which is financed by the Netherlands Organisation for Scientific Research (NWO). 
Open Access This article is distributed under the terms of the Creative Commons Attribution 4.0 International License (http:// creativecommons.org/licenses/by/4.0/), which permits unrestricted use, distribution, and reproduction in any medium, provided you give appropriate credit to the original author(s) and the source, provide a link to the Creative Commons license, and indicate if changes were made.

\section{References}

1. Gilbert, Frederic, J.N.M. Viaña, and C. Ineichen. 2018. Deflating the "DBS causes personality changes" bubble. Neuroethics. https://doi.org/10.1007/s12152-018-9373-8.

2. Schüpbach, M., M. Gargiulo, M.L. Welter, L. Mallet, C. Behar, J.L. Houeto, D. Maltete, V. Mesnage, and Y. Agid. 2006. Neurosurgery in Parkinson disease. Neurology 66: 18111816. https://doi.org/10.1212/01.wnl.0000234880.51322.16.

3. Müller, Sabine, Merlin Bittlinger, and Henrik Walter. 2017. Threats to neurosurgical patients posed by the personal identity debate. Neuroethics 10: 299-310. https://doi. org/10.1007/s12152-017-9304-0.

4. Gilbert, Frederic, Eliza Goddard, John Noel M. Viana, Adrian Carter, and Malcolm Horne. 2017. I miss being me: Phenomenological effects of deep brain stimulation. $A J O B$ Neuroscience 8: 96-109.

5. De Haan, Sanneke, Erik Rietveld, Martin Stokhof, and Damiaan Denys. 2017. Becoming more oneself? Changes in personality following DBS treatment for psychiatric disorders: Experiences of OCD patients and general considerations. PLoS ONE 12: 1-27. https://doi.org/10.1371/journal. pone. 0175748 .

6. Leentjens, Albert F.G., V. Visser-Vandewalle, Y. Temel, and F.R. Verhey. 2004. Manipulation of mental competence: An ethical problem in case of electrical stimulation of the subthalamic nucleus for severe Parkinson's disease. Nederlands Tijdschrift voor Geneeskunde 28: 1394-1398.

7. Janssen, Marcus L.F., Annelien A. Duits, Ali M. Tourai, Linda Ackermans, Albert F.G. Leentjens, Vivianne Van Kranen-Mastenbroek, Mayke Oosterloo, Veerle VisserVandewalle, and Yasin Temel. 2014. Subthalamic nucleus high-frequency stimulation for advanced Parkinson's disease: Motor and neuropsychological outcome after 10 years. Stereotactic and Functional Neurosurgery 92: 381-387. https://doi.org/10.1159/000366066.

8. Schechtman, Marya. 2010. Philosophical reflections on narrative and deep brain stimulation. The Journal of Clinical Ethics 21(2): 133-9.

9. de Haan, Sanneke, Erik Rietveld, Martin Stokhof, and Damiaan Denys. 2013. The phenomenology of deep brain stimulation-induced changes in OCD: An enactive affordance-based model. Frontiers in Human Neuroscience 7: 1-14. https://doi.org/10.3389/fnhum.2013.00653.

10. Mantione, M., D.H. Nieman, M. Figee, and D. Denys. 2014. Cognitive-behavioural therapy augments the effects of deep brain stimulation in obsessive-compulsive disorder. Psychological Medicine 44: 3515-3522. https://doi. org/10.1017/S0033291714000956.
11. Snoek, Anke. 2017. Does DBS alienate identity or does it simply fail to restore identity already eroded by illness? AJOB Neuroscience 8: 114-115. https://doi.org/10.1080 /21507740.2017.1320332.

12. Mecacci, Giulio, and Pim W.F.G. Haselager. 2017. Positive outcomes and causal insufficiency do not rule out the risk (and importance) of DBS-related identity changes. AJOB Neuroscience. https://doi.org/10.1007/978-1-59745-152-9_8.

13. Pugh, Jonathan, Laurie Pycroft, Hannah Maslen, Tipu Aziz, and Julian Savulescu. 2018. Evidence-based Neuroethics, deep brain stimulation and personality - deflating, but not bursting, the bubble. Neuroethics. https://doi.org/10.1007 /s12152-018-9392-5.

14. Baylis, Françoise. 2013. "I am who I am": On the perceived threats to personal identity from deep brain stimulation. Neuroethics. 6: 513-526. https://doi.org/10.1007/s12152011-9137-1.

15. Schermer, Maartje. 2009. Changes in the self: The need for conceptual research next to empirical research. The American Journal of Bioethics 9: 45-47.

16. Mackenzie, Catriona. 2009. Personal identity, narrative integration, and embodiment. In Embodiment and agency, eds. Sue Campbell, Letitia Meynell, and Susan Sherwin. Pennsylvania State University Press, 100-125.

17. Carel, Havi. 2008. Illness. The cry of the flesh. Stocksfield: Acumen.

18. Toombs, Kay S. 2001. Reflections on bodily change: The lived experience of disability. In Handbook of phenomenology and medicine, ed. Kay S. Toombs, 247-262. Dordrecht: Kluwer.

19. Frank, A.W. 2010. The wounded storyteller: Body, illness, and ethics. Chicago: University of Chicago Press.

20. Charmaz, Kathy, and Dana Rosenfeld. 2006. Reflections on the body, images of self: Visibility and invisibility in chronic illness and disability. In Body/embodiment: Symbolic interaction and the sociology of the body, ed. D.D. Waskul and P. Vannini, 35-51. Hampshire: Ashgate.

21. Dubiel, Helmut. 2006. Tief im Hirn. München: Kunstmann.

22. Schermer, Maartje. 2009. The mind and the machine. On the conceptual and moral implications of brain-machine interaction. NanoEthics. 3: 217-230. https://doi.org/10.1007 /s11569-009-0076-9.

23. Hansson, Sven Ove. 2005. Implant ethics. Journal of Medical Ethics 31: 519-525. https://doi.org/10.1136 /jme.2004.009803.

24. Harlow, John M. 1868. Recovery from the passage of an iron bar through the head. Massachusetts Medical Society 2: 122. https://doi.org/10.1300/J233v04n01_06.

25. Nyholm, Sven. 2018. Is the personal identity debate a "threat" to neurosurgical patients? A reply to Müller et al. Neuroethics 11: 229-235. https://doi.org/10.1007/s12152017-9337-4.

26. Nyholm, Sven, and Elizabeth O'Neill. 2016. Deep brain stimulation, continuity over time, and the true self. Cambridge Quarterly of Healthcare Ethics 25: 647-658. https://doi.org/10.1017/s0963180116000372.

27. Gisquet, Elsa. 2008. Cerebral implants and Parkinson's disease: A unique form of biographical disruption? Social Science and Medicine 67. Elsevier Ltd: 1847-1851. https://doi.org/10.1016/j.socscimed.2008.09.026. 
28. Gilbert, Frederic. 2012. The burden of normality: From "chronically ill" to "symptom free". New ethical challenges for deep brain stimulation postoperative treatment. Journal of Medical Ethics 38: 408-412. https://doi.org/10.1136 /medethics-2011-100044.

29. Chang, Chun-Hung, Shin-Yuan Chen, Yi-Ling Hsiao, Sheng-Tzung Tsai, and Hsin-Chi Tsai. 2010. Hypomania with hypersexuality following bilateral anterior limb stimulation in obsessive-compulsive disorder. Journal of Neurosurgery 112: 1299-1300. https://doi.org/10.3171 /2009.10.JNS09918.

30. Denys, Damiaan, Mariska Mantione, Martijn Figee, Pepijn Van Den Munckhof, Frank Koerselman, Herman Westenberg, Andries Bosch, and Rick Schuurman. 2010. Deep brain stimulation of the nucleus accumbens for treatment-refractory obsessive-compulsive disorder. Archives of General Psychiatry 67: 1061-1068. https://doi. org/10.1001/archgenpsychiatry.2010.122.

31. Schermer, Maartje. 2014. The cyborg-fear: How conceptual dualisms shape our self-understanding. AJOB Neuroscience 5: 56-57. https://doi.org/10.1080/21507740.2014.951784.

32. Schermer, Maartje. 2011. Ethical issues in deep brain stimulation. Frontiers in Integrative Neuroscience 5. https://doi. org/10.3389/fnint.2011.00017.

33. de Haan, Sanneke. 2017. Missing oneself or becoming oneself? The difficulty of what "becoming a different person" means. AJOB Neuroscience 8: 110-112. https://doi. org/10.1080/21507740.2017.1320330.
34. Agid, Y., M. Schüpbach, M. Gargiulo, L. Mallet, J.L. Houeto, C. Behar, D. Maltête, V. Mesnage, and M.L. Welter. 2006. Neurosurgery in Parkinson's disease: The doctor is happy, the patient less so. Journal of Neural Transmission [Suppl: 409-414. https://doi.org/10.1007 1978-3-211-45295-0_61.

35. Nuffield Council on Bioethics. 2013. Novel neurotechnologies : Intervening in the brain. In Intervening in the brain : A guide to the report. London: Nuffield Council on Bioethics. p.1-298. http://nuffieldbioethics.org/wp-content/uploads/2013/06 Novel_neurotechnologies_report_PDF_web_0.pdf. Accessed 12 Dec 2018.

36. Bluhm, Robyn, and Laura Y Cabrera. 2018. It 's not just counting that counts : A reply to Gilbert, Viaña, and Ineichen. Neuroethics https://doi.org/10.1007/s12152-0189391-6

37. Strech, Daniel. 2010. How factual do we want the facts? Criteria for a critical appraisal of empirical research for use in ethics. Journal of Medical Ethics 36: 222-225. https://doi. org/10.1136/jme.2009.033225.

38. Horstkötter, Dorothee, and Guido De Wert. 2019. Deep brain stimulation; ethical considerations. In Managing deep brain stimulation - an interdisciplinary approach, ed. \&. A. F. G. Leentjens Y. Temel, S. Chabardes, R. M. A. de Bie, J. Volkmann.

Publisher's Note Springer Nature remains neutral with regard to jurisdictional claims in published maps and institutional affiliations. 